\title{
РАЗРАБОТКА МЕТОДИКИ ОПРЕДЕЛЕНИЯ ПРОСТРАНСТВЕННОГО ПОЛОЖЕНИЯ ОБЪЕКТОВ НЕДВИЖИМОСТИ
}

\section{Евгений Ильич Аврунев}

Сибирский государственный университет геосистем и технологий, 630108, Россия, г. Новосибирск, ул. Плахотного, 10, кандидат технических наук, директор Института кадастра и природопользования, тел. (383)344-31-73, e-mail: kadastr204@yandex.ru

\section{Павел Сергеевич Батин}

ООО «Геосити», 630054, Россия, г. Новосибирск, ул. Титова 29/1, директор, тел. (903)999-38-26, e-mail: batin866@mail.ru

\section{Николай Олегович Гревцев}

Сибирский государственный университет геосистем и технологий, 630108, Россия г. Новосибирск, ул. Плахотного, 10, обучающийся, тел. (960)797-32-33, e-mail: kadastr204@yandex.ru

В статье предложена методика определения пространственного положения объектов недвижимости, заключающаяся в построении линейно-угловой сети, относительно которой определяются плановые координаты, заложенных в тело контролируемого объекта деформационных знаков. Для повышения точности уравненных координат предложено использовать два электронных тахеометра. В результате сравнения полученных результатов установлена реальная точность пространственного положения деформационных знаков и предложен статистический критерий для определения значимости планового перемещения контролируемого инженерного сооружения. Установлено, что применение при геодезических измерениях двух электронных тахеометров увеличивает точность уравненных параметров примерно на сорок процентов.

Ключевые слова: объект недвижимости, геодезический мониторинг, координаты, деформация, средняя квадратическая ошибка

\section{DEVELOPMENT OF A METHOD FOR DETERMINING SPATIAL POSITION OF CAPITAL CONSTRUCTION OBJECTS}

\section{Evgeny I. Avrunev}

Siberian State University of Geosystems and Technologies, 10, Plakhotnogo St., Novosibirsk, 630108, Russia, Ph. D., Director of the Institute of Cadastre and Environmental Management, phone: (383)344-31-73, e-mail: kadastr204@yandex.ru

\section{Pavel S. Batin}

LLC «Geocity», 29/1, Titova St., Novosibirsk, 630054, Russia, Director, phone: (903)999-38-26, e-mail: batin866@mail.ru

\section{Nikolai O. Grevtsev}

Siberian State University of Geosystems and Technologies, 10, Plakhotnogo St., Novosibirsk, 630108, Russia, Student, phone: (960)797-32-33, e-mail: kadastr204@yandex.ru

The article proposes a method of determining the spatial position of real estate, which consists of the construction of a linear-corner network, according to which the planned coordinates laid in 
the body of the controlled object of deformation marks are determined. Two electronic total stations are used to improve the accuracy of the equalized coordinates. The comparison of the results, the real accuracy of the spatial position of the deformation marks is established and a statistical criterion is proposed to determine the significance of the movement of the controlled engineering facility. It has been established that the use of two electronic total stations for geodesic measurements increases the accuracy of the equalized parameters by about forty percent.

Keywords: capital construction object, geodesic monitoring, coordinates, deformation, average square error

\section{Введение}

Решение многих задач геодезического мониторинга объектов недвижимости, расположенных в соответствующем территориальном образовании, обусловливает необходимость их пространственного определения. Результаты геодезического мониторинга необходимы для проведения соответствующих профилактических мероприятий при развитии деформационного процесса, когда зданиям и инженерным сооружениям, расположенным в зонах техногенного и антропогенного воздействия, угрожает опасность серьезного повреждения или полного разрушения. Кроме этого определение пространственного местоположения объектов недвижимости необходимо для формирования единого геопространства территориального образования и на его основе создания кадастра недвижимости в формате 3D [1-4].

\section{Постановка проблемь}

Решение поставленной научно-технической задачи осуществляется в результате выполнения геодезических измерений и вычислении координат соответствующих точек, фиксирующих на местности местоположение объекта недвижимости в пространственной $(\mathrm{X}, \mathrm{Y}, \mathrm{Z})$ или плоской прямоугольной координатной системе $(\mathrm{x}, \mathrm{y}, \mathrm{H})$. Повторение через определенный интервал времени $\Delta \mathrm{t}$ геодезических измерений и повторное вычисление координат деформационных знаков позволяет определить деформации объекта недвижимости и оценить возможность его безопасного функционирования с соответствующей корректировкой кадастровой стоимости.

Существующие и широко применяемые в настоящее время многочисленные методики определения местоположения объектов недвижимости достаточно подробно отработаны в области установления высотной составляющей (координаты $\mathrm{Z}$ или $\mathrm{H}$ ), чего нельзя сказать о плановых координатах, которые позволяют обеспечить проведение деформационного мониторинга в пространстве.

В кадастровых работах для определения планового положения объекта недвижимости используются методики, позволяющие определять плановое положение $(\mathrm{x}, \mathrm{y})$ с нормативной точностью $\mathrm{m}=5$ см, которой недостаточно для проведения деформационного мониторинга. 
Поэтому представляется актуальным разработать методику пространственного положения объекта недвижимости исходя из нормативных требований к определению плановых деформаций инженерного сооружения.

\section{Алгоритм решения проблемы}

Для разработки и адаптации предложенной методики был выбран производственный объект, расположенный в городе Новосибирск на улице Семьи Шамшиных, растровое изображение которого приведено на рисунке. Выполнение работ по проведению деформационного мониторинга было обусловлено строительством нового здания, расположенного в непосредственной близости от существующих объектов недвижимости.

Для определения планового положения в пространстве контролируемых объектов недвижимости и осуществления их деформационного мониторинга был выбран метод линейно-угловой сети с использованием наземных измерительных технологий. Технологическая схема состояла из двух этапов: на первом этапе на устойчивом геологическом основании строилась исходная линейно угловая сеть, а на втором этапе с исходных пунктов этой сети способом полярных координат определялись пространственные координаты стенных деформационных знаков, заложенных в тело контролируемых инженерных сооружений, как показано на рисунке.

В качестве измерительного технологического оборудования использовались два электронных тахеометра Sokkia SET 630RK и Trimle M3DR, инструментальные точности которых для угловых измерений составляли: $\mathrm{m}_{\beta}=6$ ", a линейных $-\mathrm{m}_{\mathrm{L}}=2$ мм. Измерения выполнялись тремя приемами с предельным расхождением горизонтальных углов между приемами 10 секунд.

Поскольку измерения выполнялись двумя электронными тахеометрами, то соответственно СКО измерений вычислялись по следующей формуле

$$
m_{\beta}=\frac{m_{\beta}}{\sqrt{2}}=\frac{6^{\prime \prime}}{\sqrt{2}}=4,2^{\prime \prime} ; \quad m_{L}=\frac{m_{L}}{\sqrt{2}}=\frac{2 M M}{\sqrt{2}}=1,4 M \mathcal{M} .
$$

Структуру линейно-угловой сети было предложено составить из следующих элементов:

1. Точки полигонометрического хода (Т1-Т5), расположенные на физической поверхности земли в непосредственной близости от контролируемого объекта и закрепленные на местности металлическими штырями, позволяющие выполнить центрирование измерительного геодезического оборудования с точностью $\mathrm{m}_{Ц}=1 \mathrm{мм}$;

2. Стенные марки (t11-t18), представленные в виде светоотражающих пленок и наклеенные на стенах зданий и сооружений, расположенных на устойчивом геологическом основании и которые в последующих циклах будут использоваться как исходные при определении стабильности точек линейно-угловой сети; 
3. Деформационные знаки (M4-M15), расположенные в стенах контролируемых объектов, по перемещению которых возможно определять плановые деформации контролируемых сооружений.

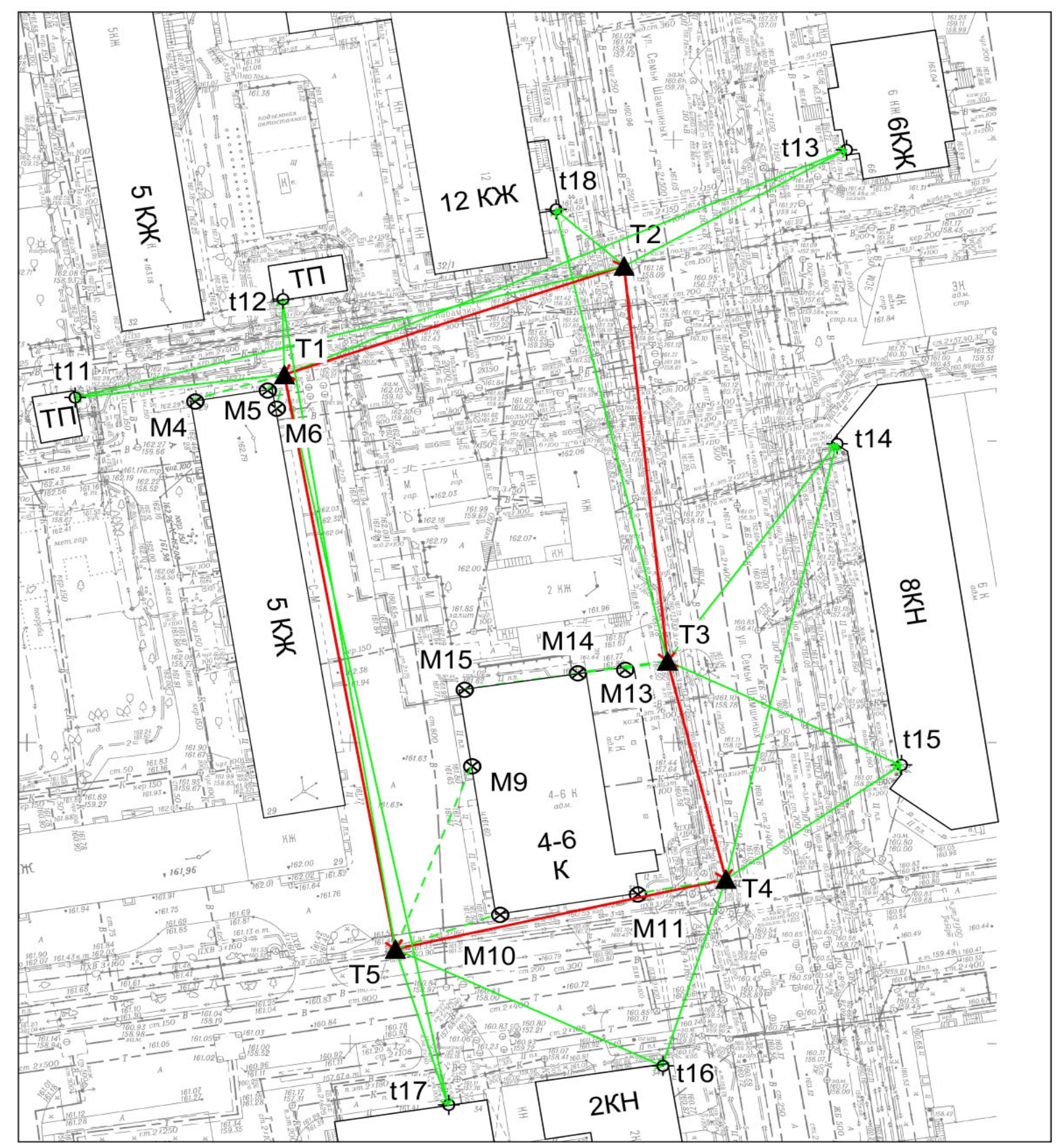

Условные обозначения:

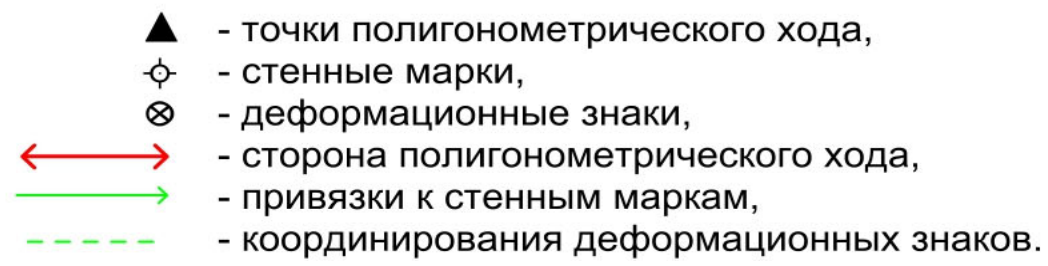

Предложенная структура линейно-угловой сети 
Уравнивание результатов измерений в линейно-угловой сети выполнялось в лицензионном программном обеспечении CREDO. В качестве исходного пункта в первом цикле наблюдений использовалась точка $\mathrm{t} 1$, а в качестве исходных дирекционных углов - направления на стенные марки t11-t13.

\section{Результаты}

Результаты уравнивания геодезических измерений в виде координат пунктов линейно-угловой сети, стенных марок и деформационных знаков отдельно по использованным электронным тахеометрам приведены в табл. 1.

Таблиия 1

Результаты уравнивания геодезических измерений

\begin{tabular}{|c|c|c|c|c|c|c|c|}
\hline \multirow{2}{*}{ № } & \multicolumn{3}{|c|}{ Используемые тахеометры } & \multicolumn{3}{c|}{ Расхождения } \\
\cline { 2 - 5 } & Sokkia SET 630RK & \multicolumn{2}{|c|}{ Trimle M3DR } & \multirow{2}{*}{$\Delta$ Х(м) } & $\Delta$ Х(м) & \multirow{2}{*}{$\Delta(\mathrm{M})$} \\
\cline { 2 - 5 } & $\mathrm{X}(\mathrm{M})$ & $\mathrm{Y}(\mathrm{M})$ & $\mathrm{X}(\mathrm{M})$ & $\mathrm{Y}(\mathrm{M})$ & & & \\
\hline M10 & 5,274 & 115,319 & 5,270 & 115,320 & 0,004 & $-0,001$ & 0,004 \\
\hline M11 & 4,523 & 136,865 & 4,516 & 136,864 & 0,007 & 0,001 & 0,007 \\
\hline M13 & 41,424 & 142,134 & 41,421 & 142,134 & 0,003 & 0,000 & 0,003 \\
\hline M14 & 42,061 & 135,798 & 42,059 & 135,798 & 0,002 & 0,000 & 0,002 \\
\hline M15 & 42,838 & 117,238 & 42,835 & 117,237 & 0,003 & 0,001 & 0,003 \\
\hline M4 & 97,553 & 84,556 & 97,554 & 84,555 & $-0,001$ & 0,001 & 0,001 \\
\hline M5 & 97,344 & 96,456 & 97,343 & 96,455 & 0,001 & 0,001 & 0,001 \\
\hline M6 & 92,299 & 97,086 & 92,298 & 97,086 & 0,001 & 0,000 & 0,001 \\
\hline T2 & 107,380 & 152,961 & 107,383 & 152,963 & $-0,003$ & $-0,002$ & 0,004 \\
\hline T3 & 41,611 & 149,372 & 41,610 & 149,373 & 0,001 & $-0,001$ & 0,001 \\
\hline T4 & 4,885 & 149,988 & 4,878 & 149,989 & 0,007 & $-0,001$ & 0,007 \\
\hline T5 & 2,291 & 100,000 & 2,287 & 100,000 & 0,004 & 0,000 & 0,004 \\
\hline t11 & 71,147 & 154,150 & 71,140 & 154,155 & 0,007 & $-0,005$ & 0,009 \\
\hline t12 & 111,403 & 100,403 & 111,402 & 100,403 & 0,001 & 0,000 & 0,001 \\
\hline t13 & 114,715 & 193,322 & 114,719 & 193,324 & $-0,004$ & $-0,002$ & 0,004 \\
\hline t14 & 72,626 & 180,578 & 72,621 & 180,578 & 0,005 & 0,000 & 0,005 \\
\hline t15 & 17,124 & 180,779 & 17,120 & 180,781 & 0,004 & $-0,002$ & 0,004 \\
\hline t16 & $-23,948$ & 136,553 & $-23,956$ & 136,552 & 0,008 & 0,001 & 0,008 \\
\hline t17 & $-24,629$ & 103,023 & $-24,633$ & 103,021 & 0,004 & 0,002 & 0,004 \\
\hline t18 & 118,301 & 146,309 & 118,304 & 146,309 & $-0,003$ & 0,000 & 0,003 \\
\hline
\end{tabular}

Анализ полученных расхождений выполнялся с использованием следующей формулы Гаусса

$$
m=\frac{\sum_{i=1}^{n} \Delta^{2}}{n},
$$

где $\mathrm{n}$ - число оцениваемых элементов.

На основании формулы (2) точность определения координат пунктов линейно-угловой сети и деформационных знаков составляет $\mathrm{m}=0,003$ м, а точность определения стенных марок соответственно $-\mathrm{m}=0,004$ м. 
Плановое перемещение деформационного знака может считаться установленным только в том случае, когда выполняется следующий статистический критерий

$$
D \geq t \cdot m_{D}=2 \cdot \Delta=2 \cdot 0,003 \text { MM }=0,006 \mathrm{MM},
$$

где $\mathrm{t}$ - статистический коэффициент, зависящий от доверительной вероятности оценивания вычисленного перемещения (при $\beta=95 \%, \mathrm{t}=2$ );

$\mathrm{D}$ - вычисленное из дух циклов геодезических наблюдений плановое перемещение деформационного знака.

Следовательно, при реализации такого способа определения плановых деформаций, движение деформационного знака может быть установлено только в том случае, когда оно превосходит 6 мм.

Следует отметить, что результаты оценки точности, полученные при математической обработке измерений и приведенные в табл. 2, дают завышенные результаты в среднем на сорок процентов.

Таблииа 2

Оценка точности определения координат, полученные в результате уравнивания линейно-угловой сети по программе CREDO

\begin{tabular}{|c|c|c|c|}
\hline Пункт & $\mathrm{m}$ & $\mathrm{mx}_{\mathrm{X}}$ & $\mathrm{m}$ \\
\hline $\mathrm{T} 2$ & 0,006 & 0,002 & 0,006 \\
\hline $\mathrm{T} 3$ & 0,008 & 0,006 & 0,006 \\
\hline $\mathrm{T} 4$ & 0,009 & 0,006 & 0,006 \\
\hline $\mathrm{T} 5$ & 0,006 & 0,006 & 0,000 \\
\hline $\mathrm{t} 11$ & 0,010 & 0,001 & 0,010 \\
\hline $\mathrm{t} 12$ & 0,010 & 0,010 & 0,001 \\
\hline $\mathrm{t} 13$ & 0,011 & 0,004 & 0,010 \\
\hline $\mathrm{t} 14$ & 0,012 & 0,009 & 0,008 \\
\hline $\mathrm{t} 15$ & 0,010 & 0,006 & 0,007 \\
\hline $\mathrm{t} 16$ & 0,009 & 0,007 & 0,005 \\
\hline $\mathrm{t} 17$ & 0,010 & 0,010 & 0,002 \\
\hline $\mathrm{t} 18$ & 0,009 & 0,006 & 0,007 \\
\hline
\end{tabular}

Таким образом, при использовании двух электронных тахеометров полученные результаты оценки точности следует уменьшать на значение 1,4. 


\section{Заключение}

В результате выполненных теоретических и экспериментальных исследований следует сделать следующие выводы:

1. Применение при геодезических измерениях двух электронных тахеометров обеспечивает увеличение точности определяемых параметров (координат деформационных знаков) на величину 1,4 ;

2. Предложенная методика определения пространственного положения объектов недвижимости позволяет получить точность плановых координат деформационных знаков $\mathrm{m}=3$ мм, следовательно, деформации в плане инженерного сооружения могут быть обнаружены только в том случае, когда они превосходят $\mathrm{D}=6 \mathrm{мм}$;

3. При требовании заказчика о повышении точности определения плановых деформаций объекта недвижимости, необходимо использовать тахеометры более высокой инструментальной точности.

\section{БИБЛИОГРАФИЧЕСКИЙ СПИСОК}

1. Аврунев Е.И. Технологические аспекты построения 3D-модели инженерных сооружений в городах арктического региона РФ [Текст] /, А. В. Чернов, А. В. Дубровский, А. В. Комиссаров, Е. Ю. Пасечник // Известия Томского политехн. ун-та. Инжиниринг георесурсов. 2018. - T. 329, № 7. - C.131-137.

2. Карпик А. П. Анализ состояния и проблемы геоинформационного обеспечения территорий // Изв. вузов. Геодезия и аэрофотосьемка. - 2014. - № 4. - С. 3-7.

3. Николаев Н.А., Чернов А. В. Трехмерный кадастр недвижимости как новая ступень развития кадастровых систем // Интерэкспо ГЕО-Сибирь-2014 : Х Междунар. науч. конгр., 8-18 апр. 2014 г., Новосибирск; Междунар. науч. конф. "Экон. развитие Сибири и Дальнего Востока. Экономика природопользования, землеустройство, лесоустройство, управление недвижимостью": сб. материалов в 2 т. - Новосибирск : СГГА, 2014. - Т. 2. - С. 194-198.

4. Чернов А.В. Исследование вариантов построения 3D-модели объектов недвижимости для целей кадастра // Вестник СГУГиТ. - 2018 - Т. 23 (3). - С. 192-210.

5. Аврунев Е.И., Колмогоров В.Г., Новоселов Ю.А., Труханов А.Э. Инвентаризация сведений ГКН о местоположении границ земельных участков и объектов капитального строительства. // Геодезия и аэрофотосъемка Известия высших учебных заведений. - 2014. №4/C - C. 181-184.

6. Карпик А.П., Варламов А.А., Аврунев Е.И. “Совершенствование методики контроля качества спутникового позиционирования при создании геоинформационного пространства территориального образования" Геодезия и аэрофотосъемка Известия высших учебных заведений №4/c (ВАК), 2014г. - С. 185-188. 